PROCEEDINGS OF THE

AMERICAN MATHEMATICAL SOCIETY

Volume 134, Number 11, November 2006, Pages 3255-3256

S 0002-9939(06)08375-4

Article electronically published on May 8, 2006

\title{
AN ELEMENTARY PROOF OF THE CHARACTERIZATION OF ISOMORPHISMS OF STANDARD OPERATOR ALGEBRAS
}

\author{
MOHAMMAD B. ASADI AND A. KHOSRAVI
}

(Communicated by Joseph A. Ball)

\begin{abstract}
This study provides an elementary proof of the well-known fact that any isomorphism $\pi: \mathcal{A} \rightarrow \mathcal{B}$ of standard operator algebras on normed spaces $X, Y$, respectively, is spatial; i.e., there exists a topological isomorphism $T: X \rightarrow Y$ such that $\pi(A)=T A T^{-1}$ for any $A \in \mathcal{A}$. In particular, $\pi$ is continuous.
\end{abstract}

Paul R. Chernoff has shown that isomorphisms of standard operator algebras are spatial in the sense explained below. An elementary proof of this theorem is given in this study by an adaptation of a remark by P. R. Chernoff [1].

Let $X, Y$ be normed spaces. Denote by $B(X)$ the algebra of all bounded linear operators on $X$. A subalgebra of $B(X)$ which contains $F(X)$ (the ideal of all finite rank operators in $B(X))$ is called a standard operator algebra on $X$.

The dual space of $X$ is denoted by $X^{*}$. If $x \in X$ and $f \in X^{*}$, then $x \otimes f$ denotes the operator defined by

$$
(x \otimes f)(z)=f(z) x, \text { for any } z \in X .
$$

If $A: X \rightarrow Y$ is a bounded linear operator, then $A^{*}: Y^{*} \rightarrow X^{*}$, the adjoint of $A$, is defined by $A^{*}(f)=f \circ A$, for any $f \in Y^{*}$.

Theorem. Let $X$ and $Y$ be normed spaces, and let $\mathcal{A}$ and $\mathcal{B}$ be standard operator algebras on $X$ and $Y$, respectively. Then every algebraic isomorphism $\pi: \mathcal{A} \rightarrow \mathcal{B}$ is spatial; i.e., there is a linear topological isomorphism $T: X \rightarrow Y$ such that for any $A \in \mathcal{A}, \pi(A)=T A T^{-1}$. In particular, $\pi$ is continuous.

Proof. Let $P \in \mathcal{A}$ be a rank one idempotent; then $\pi(P)$ is rank one idempotent, too. Then there exists $x_{0} \in X, y_{0} \in Y$ such that $P\left(x_{0}\right)=x_{0}$ and $\pi(P)\left(y_{0}\right)=y_{0}$. Also, let $S: X \rightarrow Y$ be a linear operator such that $S\left(x_{0}\right)=y_{0}$. It is easy to see that there are linear isomorphisms $\psi_{1}: \mathcal{A} P \rightarrow X$ and $\psi_{2}: \mathcal{B} \pi(P) \rightarrow Y$ such that $\psi_{1}(A P)=A P x_{0}$ and $\psi_{2}(B \pi(P))=B \pi(P) y_{0}$ for any $A \in \mathcal{A}, B \in \mathcal{B}$.

Hence, there exists an isomorphism $T: X \rightarrow Y$ such that for any $A \in \mathcal{A}$,

$$
T A P x_{0}=\pi(A) \pi(P) y_{0}=\pi(A) \pi(P) S x_{0} .
$$

Therefore $T A P=\pi(A) \pi(P) S P$, and so for any $A^{\prime} \in \mathcal{A}$,

$$
T A A^{\prime} P=\pi(A) \pi\left(A^{\prime}\right) \pi(P) S P=\pi(A) T A^{\prime} P .
$$

Received by the editors May 13, 2005 and, in revised form, May 18, 2005.

2000 Mathematics Subject Classification. Primary 47B49.

Key words and phrases. Standard operator spaces, dual space, bounded operator.

(C)2006 American Mathematical Society 
It follows that

$$
T A=\pi(A) T \text {. }
$$

To see that $T$ is bounded, let $0 \neq f \in Y^{*}$ and $0 \neq y \in Y$. Since $\pi$ is surjective, there exists an $A \in \mathcal{A}$ such that $y \otimes f=\pi(A)=T A T^{-1}$. This implies $A=$ $T^{-1}(y \otimes f) T=T^{-1}(y) \otimes(f \circ T) \in \mathcal{A}$. Therefore $T^{-1}(y) \otimes(f \circ T)$ is bounded and hence $f \circ T$ is bounded.

Then, $f \rightarrow f \circ T$ (which is the same as $T^{*}$ ) is a linear transformation from $Y^{*}$ into $X^{*}$.

Suppose $f_{n} \rightarrow 0$ in $Y^{*}$ and $T^{*} f_{n} \rightarrow g$ in $X^{*}$. Clearly, $\pi(x \otimes g)^{*} \in F\left(Y^{*}\right)$, for any $x \in X$. Therefore, $(x \otimes g)^{*} T^{*} f_{n} \rightarrow 0$ by $(*)$. But $(x \otimes g)^{*}\left(T^{*} f_{n}\right) \rightarrow(x \otimes g)^{*} g$. This implies $g=0$. By the closed graph theorem $T^{*}$ is bounded, and this implies that $T$ is bounded.

Remark. We note that in our proof, we did not use the completeness of $X$ and $Y$; so, many similar results in the literature (e.g., 2], 4], [5]), which use Banach spaces, can be generalized to normed spaces, using the above method.

\section{ACKNOWLEDGMENT}

The authors would like to thank the referee for his useful comments.

\section{REFERENCES}

[1] P. R. Chernoff, Representations, automorphisms, and derivations of some operator algebras, J. of Functional Analysis 12 (1973), 275-289. MR0350442 (50:2934)

[2] Ali A. Jafarian and A. R. Sourour, Spectrum-preserving linear maps, J. of Functional Analysis 66 (1986), 255-261. MR0832991 (87m:47011)

[3] W. Rudin, Functional Analysis, McGraw-Hill, 1991. MR.1157815 (92k:46001)

[4] Peter Šemrl, Applying projective geomery to transformations on rank one idempotents, J. of Functional Analysis 210 (2004), 248-257. MR2052121 (2005a:47063)

[5] Peter Šemrl, Invertibility preserving linear maps and algebraic reflexivity of elementary operators of length one, Proc. Amer. Math. Soc. 130 (2001), 769-772. MR1866032 (2002g:47075)

Faculty of Mathematical Sciences and Computer Engineering, Teacher Training University, 599 Taleghani Avenue, Tehran 15614, Iran

E-mail address: mb.asadi@gmail.com

Faculty of Mathematical Sciences and Computer Engineering, Teacher Training University, 599 Taleghani Avenue, Tehran 15614, Iran

E-mail address: khosravi@saba.tmu.ac.ir 